\title{
A Study of Hepatitis E in Pregnancy: Maternal and Fetal Outcome
}

\author{
Gowri Sayi Prasad $^{1} \cdot$ Sayi Prasad $^{2} \cdot$ Ashok Bhupali $^{2} \cdot$ Ajit N. Patil $^{1} \cdot$ Kanishtha Parashar $^{1}$
}

Received: 16 June 2015/ Accepted: 10 July 2015/Published online: 22 August 2015

(C) Federation of Obstetric \& Gynecological Societies of India 2015

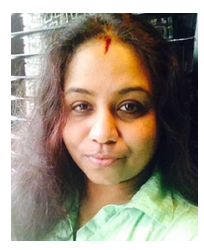

\begin{abstract}
About the Author
Dr. Gowri Sayi Prasad is an alumnus of St. John's National Academy Of Medical Sciences, Bangalore. She is a university rank holder during her postgraduation and has undergone training in Kidwai Memorial Institute of Oncology, Bangalore, in Gynecologic oncology. Currently, she is working as an Associate Professor in the Dept. of OBGY, Dr. D. Y. Patil Medical College, Kolhapur, and her strong inclination is toward high-risk obstetric care and operative gynec oncology. She is a joint author of a well-received book, Obstetric Protocols (2012). She has various publications in national journals. A recent article on Peripartum Cardiomyopathy was appreciated by leading cardiologists internationally. Her original work is on redesigning the obstetric ICU and on predicting the severity of pre-eclampsia. As a teacher, she looks forward with hope to the future by means of guiding her postgraduate students to achieve excellence in their chosen fields.
\end{abstract}

\begin{abstract}
Purpose HEV infection, a major public health concern, is known to cause large-scale epidemic and sporadic cases of acute viral hepatitis in developing countries. The infection occurs primarily in young adults and is generally mild and self-limiting; however, the case fatality rate is reportedly
\end{abstract}

higher among women, especially during the second or third trimesters of pregnancy.

Methods This study, a prospective observational study, was conducted at the Dr. D. Y. Patil Medical College Hospital and Apple Saraswati Multispeciality hospital, in Kolhapur for over a period of 3 years (Jan 2010 to Jan
Dr. Gowri Sayi Prasad is a Assoc. Prof of Obstetrics and Gynaecology in the Dr. D. Y. Patil Hospital and Research Center, Kadamwadi, Kolhapur; Sayi Prasad is the Chief Intensivist in the Apple Saraswati Multispeciality Hospital and Research Center, Kolhapur; Ashok Bhupali is the Chief Interventional Cardiologist in the Apple Saraswati Multispeciality Hospital and Research Center, Kolhapur; Ajit N. Patil is a Professor and HOD in the Department of Obstetrics and Gynaecology, Dr. D. Y. Patil Hospital and Research Center, Kolhapur; Kanishtha Parashar is a postgraduate student in the Department of Obstetrics and Gynaecology, Dr. D. Y. Patil Hospital and Research Center, Kolhapur.
$凶$ Gowri Sayi Prasad sayi_prasad@rediffmail.com

1 Department of Obstetrics and Gynaecology, Dr. D. Y. Patil Hospital and Research Center, Kadamwadi, Kolhapur, Maharashtra 416003, India

2 Apple Saraswati Multispeciality Hospital and Research Center, Kolhapur, Maharashtra, India 
2013) to find out the prevalence and clinical outcome in a series of HEV-infected pregnant women.

Results A total of fifty-five symptomatic Anti-HEV IgMpositive women were included, and the maternal-fetal outcome was analyzed. The maternal mortality was $5 \%$ including one antenatal death. Prematurity $(80 \%)$ and PROM $(11 \%)$ were the commonest fetal complications noted with a vertical transmission rate of $28 \%$.

Conclusion Variations in maternal morbidity and mortality between different studies indicate a need to subtype the viral genotype according to its virulence and morbidity.

Keywords Hepatitis E · Pregnancy ·

Fulminant hepatic failure - Maternal mortality .

Perinatal morbidity

\section{Introduction}

Hepatitis E is a single-stranded RNA virus that causes large-scale epidemic and sporadic cases of acute viral hepatitis in developing countries [1]. HEV infection, a major public health concern, is primarily transmitted through the fecal-oral route. The incubation period after exposure ranges from 3 to 8 weeks (mean 40 days) and is dose dependent $[2,3]$. The infection primarily occurs in young adults and is generally mild and self-limiting; however, the mortality rate is higher among women, especially during the second or third trimesters of pregnancy [4-6]. The incidence and severity during pregnancy vary widely around the world. In Western Europe and North America, the incidence is as low as one in 20,000, whereas in outbreaks of waterborne Hepatitis E in India and Asia, the case fatality rate is $1-2 \%$ and up to $10-20 \%$ in pregnant women [7]. Reason for the difference in the outcome of HEV in different geographical areas remains unclear [8] but could be due to early childhood HEV exposures, producing long-lasting immunity and/or modifying subsequent responses to exposure to the virus. HEV is known to have five genotypes, four of which have been detected in humans; genotypes 1 and 2 are more virulent, genotypes 3 and 4 are more attenuated and accountable for subclinical infections [7].

This disease presents a challenging situation to the obstetrician because of the complications such as antepartum hemorrhage (APH), postpartum hemorrhage ( $\mathrm{PPH})$, preterm labor, preterm premature rupture of membrane (PPROM), maternal coagulopathy, and intrauterine fetal death (IUFD).

This study was conducted jointly by the Department of Obstetrics/Gynaecology of Dr. D. Y. Patil Medical College and Hospital, Kolhapur, and Apple Saraswati Multispeciality Hospital, Kolhapur, to find out the prevalence and clinical outcome in a series of symptomatic HEV-infected pregnant women.

\section{Materials and Methods}

\section{Sample Design}

For the present research, the following methodology was adopted. A prospective observational study was conducted at the Dr. D. Y. Patil Medical College Hospital and Apple Saraswati Multispeciality hospital, a 250-bedded tertiary care center in Kolhapur, for over a period of 3 years (Jan 2010 to Jan 2013). All pregnant women presenting at any gestational age with icterus were systematically assessed for hepatitis virus infection by liver function tests and serologic analysis. The serum was analyzed for IgM AntiHEV by Rapid Immuno chromatographic Assay (Insight Device), and only Anti-HEV IgM-positive women were included in our study. Patients with clinical evidence of jaundice due to other causes, e.g., HELLP syndrome, Hemolysis, Acute Fatty Liver of Pregnancy, Biliary tract disorders, and Drug induced hepatitis, were excluded. None of the patients had a history suggestive of chronic liver disease. Maternal features such as gestational age at the time of first detection of infection, clinical progression of the disease, worsening or otherwise of laboratory parameters, and obstetric outcomes were noted in detail.

These patients were observed for viral hepatitis symptoms such as fever, edema, ascites, paralytic ileus, nasal and gastrointestinal hemorrhage, level of consciousness, and altered sensorium. Fetal well-being was monitored by ultrasonography and CTG. Further evaluation was done on an individualized basis. All patients were managed by a team of obstetricians, physicians, intensivists, and neonatologists. Patients with fulminant hepatic failure (FHF) were managed in the ICU and monitored for medical and obstetric complications. Fulminant hepatic failure was defined as the rapid development of acute liver injury with severe impairment of the synthetic function and hepatic encephalopathy in a patient without obvious, previous liver disease within 4 weeks $[9,10]$. Hepatic encephalopathy, a spectrum of neuropsychiatric abnormalities in patients with liver failure, after exclusion of other known brain diseases includes personality changes, intellectual impairment, and reduced levels of consciousness [11]. Treatment included received antibiotics, parenteral nutrition, and ventilator support, as required. Patients with deranged coagulation profile and bleeding episodes were transfused blood and blood products as deemed necessary.

All these patients were admitted and were studied regarding pregnancy status, mode of termination, and complications encountered. The decision for termination of 
pregnancy was taken on an individualized basis. Labor monitoring was conducted as per protocol. Termination of pregnancy was considered for hepatitis per se. A cord blood sample was sent in the later part of the study in the last 32 patients following a suggestion from the review committee at the end of 1 year. All the in-hospital deliveries were attended by the on-call neonatologist and appropriate care was instituted. In accordance with the institutional ethical committee norms, this study did not require a documented informed consent of the included study subjects as it did not involve any trial of intervention, and a standard treatment protocol was followed for all. Further, strict anonymity has been maintained during analysis.

\section{Results}

\section{Demographic and Obstetric Characteristics}

This study yielded the following results. Data from all 55 patients with HEV IgM antibody who were enrolled in the study were procured. The mean age of the patient at presentation of illness was $23.85 \pm 2.9$ SD years. There was no significant difference with regard to the parity status (Table 1). The majority of them were semiliterate housewives belonging to low socioeconomic status. $92 \%$ of the women had presented with symptoms of viral hepatitis for the first time in the third trimester (Table 2); the mean gestation age at presentation of symptoms was $32.6 \pm 3.37$ SD weeks.

\section{Investigation Parameters}

Hemoglobin was between 60 and $140 \mathrm{~g} / \mathrm{l}$ with a mean value of $104 \mathrm{~g} / \mathrm{l}$. The median leukocyte count was $11 \times 10^{9}$ cells $/ 1$ with the range of $2.6-28$ cells $\times 10^{9} / 1$. Platelets ranged between 28 and $441 \times 10^{9} / 1$ with a mean of $255 \times 10^{9} / 1$. Prothrombin time was between 14 and $52.5 \mathrm{~s}$ with a median value of $18 \mathrm{~s}$. Median INR was observed to be 1.8. Serum bilirubin of these patients varied between 1 and $20.4 \mathrm{mg} / \mathrm{dl}$ with a mean of $7.85 \mathrm{mg} / \mathrm{dl}$.

Table 1 Age and parity distribution of patients

\begin{tabular}{lcrll}
\hline Age (years) & Parity & \multicolumn{2}{l}{ Total } \\
\cline { 2 - 4 } & Primi & G2 & G3 or more & \\
\hline $20-25$ & 25 & 12 & 3 & $40 / 55(72.72 \%)$ \\
$26-30$ & 2 & 4 & 7 & $13 / 55(23.63 \%)$ \\
$30-40$ & 1 & 1 & - & $2 / 55(3.63 \%)$ \\
Total & 28 & 17 & 10 & 55 \\
\hline
\end{tabular}

Table 2 Patients characteristics

\begin{tabular}{ll}
\hline Trimester & Number $(\%)$ \\
\hline Second & $4(7.27)$ \\
Third & $51(92.72)$ \\
\hline Lab parameters & Present study \\
\hline Mean hemoglobin level $(\mathrm{g} / \mathrm{l})$ & 104 \\
Median leukocyte count $\left(\mathrm{cells} \times 10^{9} / \mathrm{l}\right)($ range) & $11(2.6-28)$ \\
Mean platelet count $\left(\mathrm{cells} \times 10^{9} / \mathrm{l}\right)$ & 255 \\
Mean serum bilirubin level $(\mathrm{mg} / \mathrm{dl})$ & 7.85 \\
Median SGPT $(\mathrm{U} / \mathrm{L})$ & $580(60-3800)$ \\
Median prothrombin time $(\mathrm{control} 15 \mathrm{~s})($ range) & $18(14-52.5)$ \\
Median international normalized ratio (range) & $1.8(1-3.9)$ \\
Mean serum albumin level $(\mathrm{g} / \mathrm{l})$ & 32
\end{tabular}

SGPT ranged between 60 and $3800 \mathrm{IU} / \mathrm{l}$ with a median value of $580 \mathrm{IU} / 1$.

\section{Obstetric Outcome}

Antenatal complications included preterm labor seen in 38 women $(77 \%)$ and premature rupture of membranes in 6 women (11\%) (Table 3).

Of the 55 women, 43 had a spontaneous onset of labor, three were induced, and eight underwent an emergency LSCS $(14 \%)$. The indication for induction was severe oligohydramnios and fetal distress (Table 3). All the

Table 3 Obstetric complications and intervention

\begin{tabular}{ll}
\hline Obstetric complications & Present study \\
\hline PPH & $4 / 54(7.40 \%)$ \\
Second trimester & - \\
Third trimester & $4 / 50(8 \%)$ \\
PROM & $6 / 54(11.11 \%)$ \\
Second trimester & $1 / 4(25 \%)$ \\
Third trimester & $5 / 50(10 \%)$ \\
IUFD & $5 / 55(9.09 \%)$ \\
Second trimester & $1 / 4(25 \%)$ \\
Third trimester & $4 / 50(8 \%)$ \\
Preterm labor & $38 / 49(77.55 \%)$ \\
Second trimester & $2 / 4(50 \%)$ \\
Third trimester & $36 / 45(80 \%)$ \\
Mode of delivery & \\
LSCS & $8 / 54(14.81 \%)$ \\
Third trimester & 8 \\
Vaginal delivery & $46 / 54(85.18 \%)$ \\
Second trimester & 4 \\
Third trimester & 42 \\
\hline
\end{tabular}


Table 4 Maternal mortality and medical complications

\begin{tabular}{lc}
\hline Maternal complications & The present study $(\%)$ \\
\hline Hepatic encephalopathy with FHF & $5 / 55(9.09)$ \\
Second trimester & $2 / 4(50)$ \\
Third trimester & $3 / 51(5.88)$ \\
Gastrointestinal hemorrhage & $7 / 55(12.72)$ \\
Ascites & $5 / 55(9.09)$ \\
Renal failure & $8 / 55(14.54)$ \\
Coagulation defect & $18 / 55(32.72)$ \\
ICU admission & $10 / 55(18.18)$ \\
Transfusion (blood/blood products) & $18 / 55(32.72)$ \\
Maternal mortality (overall) & $3 / 55(5.45)$ \\
\hline
\end{tabular}

Table 5 Fetal outcome

\begin{tabular}{lc}
\hline Fetal outcome & The present study $(\%)$ \\
\hline IUD & $5 / 55(9.09)$ \\
Spontaneous abortions & $1 / 55(1.81)$ \\
Preterm babies & $38 / 47(80.85)$ \\
Still birth & $2 / 49(4.08)$ \\
Live birth & $47 / 49(95.91)$ \\
Neonatal death & $2 / 47(4.25)$ \\
Low birth weight & $27 / 47(57.44)$ \\
Meconium stained liquor & $4 / 47(8.51)$ \\
NICU admissions & $19 / 47(40.42)$ \\
\hline
\end{tabular}

patients, prior to induction and LSCS, were evaluated by a senior physician, obstetrician, and anesthetist.

\section{Mortality and Complications}

There were three maternal deaths $(5 \%)$, including one antenatal death. All three women had fulminant hepatic failure leading to encephalopathy and multiorgan dysfunction. Signs and complications frequently encountered were coagulopathy (32\%), ascites $(9 \%)$, and renal failure $(14 \%)$. Around $10 \%$ of the patients required intensive care, and $32 \%$ required blood product transfusion (Table 4 ).

\section{Fetal Outcome}

Forty-seven women with Hepatitis E delivered live babies. Perinatal outcome was analyzed only after 28 weeks. There were five intrauterine fetal deaths, two still borns, two neonatal deaths, and one second trimester abortion. Two women had progressed into third trimester and delivered healthy babies in other hospitals, and one patient had died while she was pregnant. Prematurity $(80 \%)$ and PROM $(11 \%)$ were the commonest fetal complications noted. Of the babies, $57 \%$ were low birthweight and there were two neonatal deaths (4\%) (Table 5). As mentioned earlier, cord blood samples were collected for IgM HEV in the past 2 years of the study. Out of 32 samples tested, nine samples were positive for HEV IgM antibody ( $28 \%$ ). Babies were followed up for 2 weeks post delivery. None of them developed the signs of viral hepatitis. All the women breastfed their babies.

\section{Discussion}

Hepatitis E viral infection occurring in young adults is a known phenomenon with a predisposition to pregnant women [2]. Although high mortality and morbidity of both mother and fetus have been reported, the exact mechanism still remains to be explained. Of 81 women with icterus, 55 cases were attributed to Hepatitis E $(68 \%)$ which is comparable to southern Indian Studies [12], but lower than northern Indian reported rates. The differences as well as similarities between this study and the reported cases from the above quoted Indian studies cannot be overemphasized in view of referral bias as well as small restricted samples and regional differences. Compared to all the other studies of Hepatitis E in pregnancy [13-15], the subjects of this study are young, i.e., $23.85 \pm 2.9$ SD years. Parity does not seem to play much of a role since we had almost equal distribution in both primigravidae and multigravidae. The present study shows a lower mean serum albumin, median ALT level, and an INR prothrombin time as compared to few other studies from South Asia and northern India [13-15, 20]. These were all observational studies conducted on pregnant women with Hepatitis E.

With respect to the severity of liver failure, the present study has a small incidence of fulminant hepatic failure, i.e., $5 / 55(9 \%)$, with two women in second trimester. In a very orderly study from New Delhi [15], where Hepatitis E was compared to non-Hepatitis $\mathrm{E}$ of affected women, the incidence of FHF was $55 \%$. Kumar et al. [13] have reported $33 \%$ of incidence rate of FHF in a small study of 28 patients. Variation in the FHF occurrence has previously been explored [16]. The reasons for the differences in the outcome of HEV in different geographical areas remain unclear but could be due to early childhood HEV exposures, producing long-lasting immunity and/or modifying subsequent responses to exposure to the virus. Alternatively, the virulence of HEV genotypes could vary in different geographical areas $[17,18]$. This virulence ability of genotypes might play a role in determining the viral subtype which is common in pregnancy and in epidemic outbreaks.

Obstetric complications included preterm labor 38/54 (70 \%), PROM 6/54 (11\%), and postpartum hemorrhage occurring in $4 / 54(7 \%)$. There were eight cesarean sections done for obstetric indications such as previous LSCS, transverse lie, placenta praevia, and fetal distress. The 
worsening maternal condition due to infection per se did not seem to affect the caesarean rates in our study. Subtotal hysterectomy was done during cesarean section due to placenta praevia, previous LSCS, and atonic PPH. This patient later had DIC, hepatic encephalopathy, and died on post op day 7. Induction of labor was performed for indications such as non-reactive NST and oligohydramnios in 4 patients $(9 \%)$. These outcomes in pregnancy support an oft-observed phenomenon that prognosis is best when labor occurs spontaneously earlier, irrespective of gestational age [14].

Maternal mortality occurred in three women, all of whom presented with fulminant hepatic failure in third trimester, and intrauterine death was detected at the time of admission in all of them. One of these women died antenatally. Maternal mortality was considerably lower $(5 \%)$ in our study. Rates in other north Indian and Pakistani studies vary from 26 to $41 \%$ [13-15].

As regards the fetal outcome, preterm and low birth weight formed the bulk of NICU admissions (40\%). As expected, almost all the patients with FHF had poor fetal outcome, three IUD, and one neonatal death. An interesting postulation by Khuroo et al. suggested a more severe maternal course in IUD pregnancies, probably as a result of fetal hepatitis and toxins released into maternal circulation. This is observed in our study, also, wherein all the five patients who presented with IUD at admission had severe disease, i.e., coagulopathy, and three of them subsequently died. Conversely, we suggest that the severity of metabolic disturbances in these women probably triggered the IUD rather than vice versa.

Out of 32 neonates for whom cord blood sampling was done for HEV IgM, nine tested positive for HEV IgM indicating the vertical transmission rate of $28 \%$. Out of these nine neonates, seven needed NICU admissions, although not many of them had clinically evident disease later. Vertical transmission was $100 \%$, i.e., it was detected in all 26 cases of HEV RNA-positive women by testing for HEV RNA in cord blood or newborn blood in Uduman et al.'s study [19], whereas vertical transmission was observed in only 6 of the 18 cases (33.3\%) of HEVin Kumar et al.'s study [13]. In Khuroo et al.'s study, vertical transmission was diagnosed in 25 of the 36 pregnant women, i.e., the blood collected from the baby at birth or within 2 weeks of birth (minimum incubation period of the disease) had IgM anti-HEV or HEV RNA [20]. However, the possibility of contamination of cord blood with the maternal blood could not be excluded.

\section{Conclusion}

These observed variations in maternal morbidity and mortality between studies indicate a need to subtype the viral genotype according to its virulence and morbidity which are almost exclusive to pregnancy. This could form the basis for sponsored vaccination programs in endemic areas and susceptible populations. Commercially available Hepatitis E vaccine should be studied in the people as it is most harmful to pregnant mothers.

\section{Limitations}

Primarily, our study had a moderate sample size.

We cannot deny the possibility of referral bias in our study. Icteric pregnant women and early preterm deliveries requiring NICU facility were commonly referred to our institution from primary health centers.

Furthermore, the prevalence of HEV was not studied as we had only included the HEV IgM-positive patients. Patients with minor symptoms or who were asymptomatic could have been missed on clinical evaluation and were not documented.

HEV RNA testing was not done in our study as a part of investigation which would have more accurately determined the vertical transmission rate. Follow-up of the babies for manifestation of Hepatitis was not done.

Intrauterine deaths were not investigated with HEV RNA/Autopsy which could have given us an insight into the possible etiology of the affected fetus.

Author contribution Gowri Sayi Prasad: Project development, data analysis, manuscript editing. Sayi Prasad: Data analysis, manuscript editing. Ashok Bhupali: Project development. Ajit N. Patil: Project development. Kanishtha Parashar: Data collection, manuscript writing.

\section{Compliance with Ethical Standards}

Conflict of interest The authors declare that they have no conflicts of interest.

Ethical approval The following study has been approved by the Dr. D. Y. Patil Hospital's institutional ethics committee, Kolhapur.

\section{References}

1. Khuroo MS. Acute liver failure in India (Letter). Hepatology. 1997;26:244-6.

2. Krawczynski K, Aggarwal R, Kamili S. Hepatitis E. Infect Dis Clin North Am. 2000;14:669-87. doi:10.1016/S0891-5520(05)70126-4.

3. Tsarev SA, Tsareva TS, Emerson SU, et al. Infectivity titration of a prototype strain of Hepatitis $\mathrm{E}$ virus in cynomolgus monkeys. J Med Virol. 1994;43:135-42.

4. Purcell RH, Emerson SU. Hepatitis E: an emerging awareness of an old disease. J Hepatol. 2008;48:494-503.

5. Aggarwal R. Hepatitis and pregnancy. Indian J Gastroenterol. 2007;26:3-5 
6. Benait VS, Sander V, Purikh F, et al. Outcome of acute hepatic failure due to acute Hepatitis $\mathrm{E}$ in pregnant women. Indian $\mathrm{J}$ Gastroenterol. 2007;26:6-10.

7. Kasper L, Fauci J. Acute viral hepatitis. Harrison's Princ Intern Med. 2015;2(18):2537-55.

8. Lindemann ML, Gabilondo G, Romero B, et al. Low prevalence of Hepatitis $\mathrm{E}$ infection among pregnant women in Madrid, Spain. J Med Virol. 2010;82:1666-8.

9. Gotthardt D, Riediger C, Weiss KH, et al. Fulminant hepatic failure: etiology and indications for liver transplantation. Nephrol Dial Transp. 2007;22(Suppl 8):viii5-8.

10. Vaquero J, Blei AT. Etiology and management of fulminant hepatic failure. Curr Gastroenterol Rep. 2003;5:39-47.

11. Blei AT, Córdoba J, Steindl P, et al. Hepatic encephalopathy. Am Coll Gastroenterol. 2001;96(7):1968-76 Published by Elsevier Science Inc.

12. Coimbatore Azeez R, Udayakumar N, Venkataraman J. Liver disease in pregnancy and its influence on maternal and fetal mortality: a prospective study from Chennai, Southern India. Eur J Gastroenterol Hepatol. 2008;20(4):362-4.

13. Kumar A, Beniwal M, Kar P, et al. Hepatitis $\mathrm{E}$ in pregnancy. Int $\mathrm{J}$ Gynecol Ostet. 2004;85(3):240-4.

14. Tahira Y, Hashmi HA, Aisha T. Fetomaternal outcome with Hepatitis E in pregnancy. J Coll Phys Surg Pak. 2013;23(10): $711-4$.
15. Patra S, Kumar A, Trivedi SS, et al. Maternal and fetal outcomes in pregnant women with acute Hepatitis E virus infection. Ann Intern Med. 2007;147:28-33.

16. Navaneethan U, Al Mohajer M, Shata MT. Hepatitis E and pregnancy: understanding the pathogenesis. Liver Int. 2008;28(9):1190-9.

17. Stoszek SK, Engle RE, Abdel-Hamid M, et al. Hepatitis E antibody seroconversion without disease in highly endemic rural Egyptian communities. Trans $R$ Soc Trop Med Hyg. 2006;100:89-94 Epub 2005 Oct 28.

18. Stoszek SK, Abdel-Hamid M, Saleh DA, et al. High prevalence of Hepatitis E antibodies in pregnant Egyptian women. Trans R Soc Trop Med Hyg. 2006;100:95-101 Epub 2005 Oct.

19. Kumar RM, Uduman S, Rana S, et al. Sero-prevalence and mother-to-infant transmission of Hepatitis E virus among pregnant women in the United Arab Emirates. Eur J Obstet Gynecol Reprod Biol. 2001;100(1):9-15.

20. Khuroo MS, Kamili S. Saleem Kamili. Association of severity of Hepatitis E virus infection in the mother and vertically transmitted infection in the fetus. JK Pract. 2006;13(2):70-4. 